\title{
La population irlandaise contemporaine : convergence ou singularité européenne?
}

\section{Catherine Piola}

\section{Q OpenEdition \\ 1 Journals}

\section{Édition électronique}

URL : http://journals.openedition.org/etudesirlandaises/3296

DOI : 10.4000/etudesirlandaises.3296

ISSN : 2259-8863

\section{Éditeur}

Presses universitaires de Rennes

\section{Édition imprimée}

Date de publication : 30 juillet 2013

ISBN : 978-2-7535-2673-0

ISSN : 0183-973X

\section{Référence électronique}

Catherine Piola, « La population irlandaise contemporaine : convergence ou singularité européenne

? », Études irlandaises [En ligne], 38-1 | 2013, mis en ligne le 30 juin 2015, consulté le 19 avril 2019.

URL : http://journals.openedition.org/etudesirlandaises/3296 ; DOI : 10.4000/etudesirlandaises.3296 


\title{
La population irlandaise contemporaine : convergence ou singularité européenne?
}

\author{
Catherine Piola \\ Université Dauphine - Paris 12
}

\section{Résumé}

Les deux dernières décennies ont vu la population irlandaise se transformer, s'adapter aux nouveaux modes de vie et de travail et adopter les valeurs et les comportements liés à une économie mondialisée. L'article s'intéresse à ces changements d'un point de vue démographique. À la lumière des données les plus récentes, celles du recensement de 2011, l'article évalue la nature du changement démographique en cours. La question de la convergence en matière de composition et de comportements dans le contexte européen est posée dans le cas de la population de la République. Pour y répondre, les indicateurs tels que la fécondité, l'espérance de vie, les taux de mariage et de divorce, les structures familiales ou encore les migrations sont étudiés successivement et mènent à une analyse socio-économique visant à mesurer l'uniformisation des schémas démographiques dans l'Union européenne.

Mots clés : démographie irlandaise, population irlandaise, changement démographique en Irlande, migrations, fécondité, espérance de vie, famille, mariage, divorce, identité religieuse, vieillissement.

\section{Abstract}

Irish society has changed at a fast pace over the last two decades, its population adapting to new living and working patterns and adopting different values and behaviours within today's globalised world. The article focuses on these changes from a demographic point of view. Thanks to the latest data available, largely collected in the 2011 census and 2012 regular reports, the article assesses the nature of the demographic change which has taken place. It questions whether such an evolution is making the Irish population more alike the European ones, in composition and behaviour. To do so, several key factors such as fertility, life expectancy, marriage and divorce rates, family structure as well as migrations and minorities are studied, all leading to a socio-demographic analysis attempting to measure Ireland's uniformisation within the European Union.

Keywords: Irish demography, Irish population, demographic change in Ireland, migration, fertility, life expectancy, family, marriage, divorce, religious identity, ageing.

La société irlandaise s'est rapidement transformée au cours des vingt dernières années, économiquement, socialement et culturellement; la mondialisation, l'avènement du Tigre Celtique puis sa chute ont modifié les comportements 
de chacuns, les modes de vie et de travail, les valeurs individuelles et collectives. L'objectif de cet article est de rendre compte des plus récentes évolutions démographiques de la République. L'occasion en est donnée par le recensement de 2011 qui dresse un profil quantifié de la population irlandaise en ce début de $\mathrm{XXI}^{\mathrm{e}}$ siècle. Si les clichés les plus réducteurs ont longtemps été attachés à la population irlandaise, c'est sans doute que l'image d'une population blanche, jeune, catholique et prompte à émigrer n'était pas dénuée de fondement; pourtant le profil que le recensement de 2011 offre va, à de nombreux égards, bien au-delà de telles banales constatations. Certes, l'exercice du dénombrement a ses limites, nous le verrons à l'analyse de certains critères, mais il permet de dresser un profil à une date donnée qui renseigne plus que toute autre source sur la composition, la répartition et l'évolution de la population. L'hypothèse qui sous-tend cette étude est celle de la convergence du profil démographique irlandais vers celui de l'Union européenne. Pour juger de la singularité de la population irlandaise dans le contexte européen, sont abordés dans les paragraphes qui suivent les indicateurs habituels de mesure de l'accroissement de la population ainsi que des données relevant de choix plus individuels et personnels et révélant la nature des changements de la société.

Mesurer le changement démographique exige dans un premier temps de se pencher sur l'indicateur le plus synthétique de la population étudiée : sa croissance. En 2011, forte de ses 4588252 habitants, la République d'Irlande affiche une croissance intercensitaire de $8,2 \%$. Ce taux record dans le contexte européen se maintient depuis une quinzaine d'années et trouve son explication dans l'évolution des principaux moteurs de la dynamique démographique que sont la structure par âge et la fécondité d'une part, la mortalité et l'espérance de vie d'autre part. Les flux migratoires, ayant longtemps contrecarré l'expansion de la population, ont aussi récemment contribué à cet essor, comme nous le montrerons à la fin de cette section. En 2011, l'ensemble de la population de la République est réparti en classes d'âge selon les proportions suivantes.

Tableau 1 : Population par classes d'âge dans la République en 2011, en pourcentage de la population totale

\begin{tabular}{|c|c|c|c|c|c|c|c|c|c|c|c|c|}
\hline $\begin{array}{c}\text { Classes } \\
\text { d'âge }\end{array}$ & $0-4$ & $5-9$ & $\begin{array}{c}10- \\
14\end{array}$ & $\begin{array}{c}15- \\
19\end{array}$ & $\begin{array}{c}20- \\
29\end{array}$ & $\begin{array}{c}30- \\
39\end{array}$ & $\begin{array}{c}40- \\
49\end{array}$ & $\begin{array}{c}50- \\
59\end{array}$ & $\begin{array}{c}60- \\
64\end{array}$ & $\begin{array}{c}65- \\
69\end{array}$ & $\begin{array}{c}70- \\
79\end{array}$ & $80+$ \\
\hline République & 7,7 & 7 & 6,6 & 6,1 & 14,3 & 16,5 & 13,8 & 11,3 & 4,7 & 3,8 & 5 & 2,7 \\
\hline
\end{tabular}

Sources : Données calculées à partir de This is Ireland 1, Census 2011, tableau 6A, p. 66-67

Ces données soulignent une répartition faisant la part belle aux générations les plus jeunes. En effet, en 2011, plus d'un Irlandais sur quatre est âgé de moins de 
20 ans $(27,4 \%)$. A contrario, les seniors de plus de 65 ans sont plus faiblement représentés avec $11,5 \%$ du total. Il ne s'agit en rien d'une nouveauté, la tendance existant depuis plusieurs décennies. Dans le contexte de l'Union Européenne, la population de la République continue donc de se distinguer comme l'incontestable championne de la jeunesse : les moins de quinze ans de l'Union Européenne comptent pour $15 \%$ du total, ils sont $21,3 \%$ dans la République, l'écart le plus grand étant avec l'Allemagne. De même, les seniors représentent 17,4 \% de la population européenne au total, moins de $12 \%$ en Irlande, et les seniors allemands représentent un cinquième du total de la population allemande.

Par ailleurs, l'âge médian dans la République est de 36,1 ans en 2011, soit une valeur d'environ quatre ans inférieure à la moyenne de l'Union. Pour ce qui est de la fécondité, on relève l'évolution suivante.

Tableau 2: Taux de natalité (pour mille) et indice conjoncturel de fécondité en République de 1980 à 2011

\begin{tabular}{|c|c|c|c|c|c|c|c|}
\hline Année & 1980 & 1985 & 1990 & 1995 & 2000 & 2005 & 2011 \\
\hline Natalité & 21,8 & 17,6 & 15,1 & 13,5 & 14,3 & 14,8 & 16,1 \\
\hline Fécondité & 3,23 & 2,5 & 2,12 & 1,85 & 1,90 & 1,88 & 2,06 \\
\hline
\end{tabular}

Source : Statistical Yearbook 2012, p. 67

Encore élevé au début des années 1980, le taux de natalité irlandais a baissé irrégulièrement au cours de cette décennie jusqu’à sa valeur la plus basse de 13,5\%o en 1995. Le choix de familles plus petites et le meilleur conrôle des naissances chez les couples mariés ont eu un effet notable sur la natalité. La vigueur de la natalité en 2008 (16,8\%) est remarquable en comparaison et dans le contexte européen - on note au Royaume-Uni un taux de 10,65 \%o en 2009, de 12,5\%o en France en 2011. La structure de la population explique en partie ces chiffres, les Irlandaises du baby-boom des années 1970-1980 étant arrivées en âge d'être mère. L'arrivée d'une population imigrée majoritairement en âge de procréer et aux valeurs familiales proches de celles des Irlandais a contribué à cet essor, nous y reviendrons plus avant.

Le taux de fécondité en baisse jusqu'au seuil de remplacement en 2008 (avec 2,1 enfant par femme en moyenne) et légèrement en deçà depuis, reste nettement supérieur à la moyenne européenne de 1,59 . Il révèle néanmoins une fécondité qui doit son apparente bonne santé à la structure par âge de sa population et, jusqu'en 2008, à un contexte économique favorable, celui du Tigre Celtique. On pouvait penser qu'il allait faiblir sous l'effet de la crise économique, pouvant par exemple déclencher l'émigration des populations en âge de fonder une famille, jeunes adultes irlandaises de souche comme immigrées - les naissances issues 
de mères immigrées représentent en 2011 près d'un quart des naissances. Or les données relevées en 2011 n'indiquent pas de déclin, à croire que les Irlandais et les résidents étrangers ont décidé que la situation économique n’aurait pas d’impact sur leur volonté de fonder une famille.

Notons encore en matière de natalité que la tendance à un recul de l'âge de la maternité est avéré en République, à l'instar de ce qui est observé dans les pays voisins. En 2011, la moyenne d'âge des mères est de 32 ans, elle était de 28 ans vingt ans auparavant, et la présence de mères non irlandaises, originaires des pays européens de l'élargisement de 2004 en particulier, a ralenti ce recul dans la mesure où elles sont en moyenne trois ans plus jeunes que les mères irlandaises. Ce recul de l'ensemble des mères fait revenir la République à des schémas européens dont elle s'était écartée.

Si la jeunesse et la fécondité de la population irlandaise sont encore indéniables au début du $\mathrm{XXI}^{\mathrm{e}}$ siècle, une analyse plus poussée des indicateurs de structure par âge permet de déceler par ailleurs les premiers signes de vieillissement. Le tableau 1 révèle en effet que la plus grande classe d'âge est celle des trentenaires, $16,5 \%$ de la population totale en 2011 . Le tableau 3 ci-dessous souligne l'évolution dans le temps de la part relative des classes d'âge dans la population de la République entre 1971 et 2011.

Tableau 3 : Variations des effectifs et de leur part relative par classes d'âge entre 1971 et 2011

\begin{tabular}{|l|c|c|c|c|c|c|c|c|c|}
\hline Classes d'âge & $0-9$ & $10-19$ & $20-29$ & $30-39$ & $40-49$ & $50-59$ & $60-69$ & $70-79$ & $80+$ \\
\hline Effectifs & $+7,1 \%$ & $+3,4 \%$ & $+69,5 \%$ & $+152 \%$ & $+103 \%$ & $+65,2 \%$ & $+59,5 \%$ & $+45 \%$ & $+172,4 \%$ \\
\hline Proportion & $-30 \%$ & $-33 \%$ & $+10 \%$ & $+65 \%$ & $+32,7 \%$ & $+7,6 \%$ & $+3,6 \%$ & $-5,6 \%$ & $+80 \%$ \\
\hline
\end{tabular}

Sources : Données calculées à partir de Census 1971, Vol. II, p. 1 et This is Ireland 1, Census 2011, tableau 6A, p. 66-67

Les plus fortes variations touchent les classes d'âge extrêmes : la considérable augmentation des effectifs et de la proportion des plus de 80 ans attire l'attention sur le vieillissement de la population irlandaise. Elle s'accompagne d'un recul de $63 \%$ de la proportion des moins de vingt ans alors que leurs effectifs enregistrent une croissance qui confirme le relatif dynamisme de la fécondité. La sur-représentation des trentenaires est aussi à souligner puisqu'il s'agit de la seconde plus forte augmentation, en effectifs comme en taux, au cours des quarante dernières années et l'étude des mouvements migratoires se penchera dans la section suivante sur cet effet. La silhouette de la pyramide des âges de 2011 présente donc un sommet élargi, à l'instar de ce qui est observé en Europe, et un renflement central sur une base rétrécie mais encore solide. À elles seules, la structure de la population et sa fécondité ne suffisent pas à expliquer les changements enregistrés. 
La mesure de la croissance démographique résulte aussi des indicateurs de mortalité dont l'incidence sur l'allongement moyen de la vie découle directement. La mortalité varie selon un grand nombre de facteurs liés à l'état de développement de la société, la plupart relevant du domaine médical, comme l'accès aux soins et la prévention, mais aussi à l'environnement, de vie et de travail, ou encore aux comportements privilégiés par tels ou tels groupes dans la société. La réflexion qui suit s'appuie avant tout sur l'évolution de la mortalité mais s'intéresse aussi à la morbidité et à ses déterminants.

La mortalité irlandaise affichait des taux compris entre $11 \%$ et $12 \%$ jusqu'au début des années 1970, valeur qui était comparable à celle des pays européens voisins. Depuis les années 1980, en Irlande, les taux de mortalité et de mortalité infantile ont été considérablement réduits, comme en témoigne le tableau 4.

Tableau 4: Taux de mortalité et de mortalité infantile en République (pour 1000) entre 1980 et 2011

\begin{tabular}{|c|c|c|c|c|c|c|c|}
\hline Années & 1980 & 1985 & 1990 & 1995 & 2000 & 2005 & 2011 \\
\hline Mortalité & 9,8 & 9,4 & 8,9 & 8,7 & 8,2 & 6,8 & 6,1 \\
\hline Mortalité Infantile & 10,2 & 9 & 8 & 7 & 5,62 & 5,39 & 3,85 \\
\hline
\end{tabular}

Source : http://www.cso.ie/en/statistics

En 2011, l'Irlande affiche un taux de mortalité général de 6,1\% qui est en deçà de celui de l'Union européenne de 9,7\%, position qu'elle conserverait d'ailleurs comparativement à l'Union Européenne des 15 (pré-élargissement de 2004) où le taux serait de 8,96\% \% La République doit cette bonne place en partie à un facteur intrinsèque, la structure par âge de sa population. La petite taille du groupe des seniors et sa sur-féminisation minimisent le nombre de morts dus à la vieillesse proportionnellement au reste de la population. La prospérité du pays depuis les années 1990, l'augmentation du nombre de médecins par habi$\operatorname{tant}^{1}$ - sans extension des infrastructures médicales publiques ni du nombre de lits d'hôpital -, l'effet de la prévention et de l'information, ainsi que le maintien d'un système de protection médicale ouvert à tous, bien que pas nécessairement égalitaire, sont autant de facteurs extrinsèques qui ont contribué à cette faible mortalité. En matière de mortalité infantile, les progrès ont été aussi continus que remarquables, bien que les écarts entre l'Union européenne et la République soient moins marqués, respectivement $4,3 \%$ et $3,85 \%$ en 2011 , et que les valeurs irlandaises inférieures à $5 \%$ n'aient été enregistrées que depuis 2009 .

L'étude des causes de mortalité en Irlande ne révèle pas de caractéristiques propres au pays. La nation partage ses principales pathologies avec ses voisins

1. En 2002, la République comptait 2,4 médecins pour 1000 habitants, il y en avait 3,17 en 2010. 
d'Europe du Nord et des sociétés dites développées. Les plus fréquentes causes de mortalité sont les maladies cardio-vasculaires et les cancers, qui, bien que leur nombre soit en léger recul depuis quelques années, sont encore la cause de $63 \%$ des décès en 2010; viennent ensuite les maladies du système respiratoire qui comptent pour près de $12 \%$ des décès. Hommes et femmes ne sont pas égaux face à la mort, les disparités sont particulièrement marquées dans les pathologies mais aussi dans les causes accidentelles provoquant la mort.

La corrélation entre baisse de la mortalité et allongement de l'espérance de vie n'est pas fortuite; la durée moyenne de la vie dépend pourtant aussi d'autres déterminants dont la morbidité et d'un faisceau de facteurs comportementaux qui ne seront pas détaillés ici. Parallèlement au recul de la mortalité, l'espérance de vie en Irlande s'est allongée et les gains sont remarquables au cours des trente dernières années.

Tableau 5: Espérance de vie à la naissance et à 75 ans en République de 1980 à 2010

\begin{tabular}{|l|l|c|c|c|c|c|c|c|}
\hline \multicolumn{2}{|c|}{} & 1980 & 1990 & 1995 & 2000 & 2005 & 2010 & Gain total \\
\hline \multirow{2}{*}{ à la naissance } & Hommes & 70,1 & 72,3 & 73 & 75,1 & 76,8 & 78 & $+7,9$ \\
\hline \multirow{3}{*}{} & Femmes & 75,6 & 77,9 & 78,5 & 80,3 & 81,6 & 82,7 & $+7,1$ \\
\cline { 2 - 9 } & Écart & 5,5 & 5,6 & 5,5 & 5,2 & 4,8 & 4,7 & - \\
\hline \multirow{2}{*}{75 ans } & Hommes & 7,3 & 7,8 & 8 & 8,9 & 9,8 & nc & $+2,5$ \\
\hline \multirow{3}{*}{} & Femmes & 9,1 & 10,2 & 10,4 & 11,2 & 12,1 & nc & +3 \\
\cline { 2 - 9 } & Écart & 1,8 & 2,4 & 2,4 & 2,3 & 2,3 & nc & - \\
\hline
\end{tabular}

Sources : Department of Health, Health in Ireland, Key Trends 2012, table 1.6, p. 9 et Index Mundi, [http://www. indexmundi.com/facts/ireland/life-expectancy-at-birth], consulté le 12 mai 2013

Restée inférieure aux valeurs européennes jusqu'en 2001, l'espérance de vie à la naissance des Irlandais et Irlandaises est, depuis, passée au-dessus de la moyenne de l'UE27, tout en restant proche de celle-ci. En effet, en 2011, l'espérance de vie moyenne de 80,35 ans en Irlande n'est que d'environ six mois supérieure à celle de l'Union; elle est comparable à celle du Royaume-Uni mais encore relativement éloignée des meilleures valeurs européennes de la France $(81,5)$, de l'Espagne $(81,27)$ ou encore de la Suède $(81,18)$, en particulier chez les femmes. Pourtant, sur les quinze dernières années, les avancées irlandaises ont été comparativement plus prononcées : un gain total de deux ans supérieur à celui de la France par exemple.

Les espérances de vie entre hommes et femmes sont différentes, en Irlande comme ailleurs. Le tableau 5 montre que les hommes irlandais ont légèrement comblé l'écart qui les séparait des femmes à la naissance en le réduisant à moins de cinq ans au cours des trente dernières années; à 75 ans, l'écart d'espérance de vie a peu varié entre hommes et femmes de 1990 à 2005, les Irlandaises maintenant leur propension à vivre plus longtemps. 
La mesure de l'espérance de vie sans incapacité (EVSI), intégrée aux données de l'Union européenne depuis 2005, apporte une valeur qualitative à la simple mesure de la durée moyenne de la vie. Pour une même espérance de vie, le facteur bonne ou mauvaise santé détermine non seulement le bien-être de la personne mais aussi son degré de dépendance dans la société. Il s'agit donc d'une mesure qui est amenée à jouer un rôle important dans les projections à moyen et long terme pour les États. Dans l'immédiat, l'EVSI a déjà montré que les différences entre hommes et femmes se réduisaient considérablement lorsque le facteur santé y était inclus. Ainsi, les Irlandaises vivent certes plus longtemps que les Irlandais mais leur vie en bonne santé n'est plus longue que de quelques mois. Ainsi encore, en 2008 par exemple, chez les hommes irlandais et britanniques qui partageaient la même espérance de vie, l'EVSI indiquait que les Britanniques pouvait jouir d'un an et demi supplémentaire de vie en bonne santé.

Si les hommes et femmes d'Irlande ont effectivement gagné plusieurs années de vie en moyenne lors des dernières décennies, les questions de conditions de vie et de dépendance des seniors se poseront de plus en plus, à l'État, aux communautés et aux familles. Il s'agit d'une question économique qui ne sera pas traitée ici.

En 1997, le quatrième rapport de la Commission européenne sur la santé des citoyens de l'Union ${ }^{2}$ classait la République d'Irlande, avec le Danemark et le Portugal, dans la catégorie des pays ayant le plus d'efforts à fournir en matière d'espérance de vie. Depuis, dans la République, les progrès ont été notables et semblent durables malgré l'effondrement du Tigre Celtique. Une étude détaillée de la morbidité permettrait de mieux cerner les causes de la mort, de son recul et des questions sensibles comme par exemple le taux de suicide. Il semble en effet avoir fortement augmenté au cours des dix dernières années et est très préoccupant. Certes, le taux de suicide de la République demeure inférieur à celui de l'Irlande du Nord mais il suscite de nombreuses questions, sur sa validité par exemple; des témoignages et des rapports récents laissent penser qu'aujourd'hui encore le nombre de suicides officiellement enregistrés serait inférieur à la réalité3. L'embellie économique que le pays a connue au cours des quinze dernières années a sans nul doute soutenu les progrès. Toutefois, elle a aussi entraîné dans son sillage des effets indésirables qui auront eux aussi des effets sur la durée de vie moyenne, dans le court comme le long terme, en matière de sur-consommation (alimentaire, médicamenteuse ou autre), de mal-être (stress au travail, dépression) et de cadre de vie et de travail (durée des trajets, pollution, rythme de vie, logement).

2. Commission européenne, L'État de la santé dans l'Union Européenne, Réduire les écarts de santé, Bruxelles, Commission européenne, 2003.

3. On consultera les rapports Suicide in Ireland, A National Survey, Department of Public Health, 2001, 95 p. et Samaritans, Suicide Statistics Report 2011, où la section “The Underreporting of Suicide Statistics” page 21 éclaire sur les causes de sous-estimation des nombres de suicides en Irlande. 
Troisième moteur du changement démographique, les flux migratoires ont participé de manière déterminante à l'histoire de la population du pays et continuent de le faire. Flux sortants jusque dans les années 1960 ayant un effet régulateur au sens malthusien, celui de valve de sécurité, ils allaient à l'encontre de la forte fécondité alors que la mortalité était lentement mais sûrement maîtrisée. Le cliché des Irlandais s'exilant aux quatre coins du monde n'est pas loin de la réalité d'une époque qui a connu un premier terme, de courte durée, au début des années 1970 avec le retour de certains émigrés, chocs pétroliers internationaux et brève embellie nationale aidant. Pourtant, pendant les annnées 1980, l'émigration a repris sa course et ce n'est qu'à partir de 1996 qu'un faible solde migratoire positif se dégage. Dès lors, les besoins en main-d'oeuvre du Tigre Celtique ont déclenché la première véritable vague d'immigration de la République. Il convient de définir cet afflux humain afin de déterminer de quelle manière il intervient sur la composition de l'ensemble de la population et participe à sa croissance. Identifier ces mouvements est tout d'abord une question d'effectifs et de solde migratoire comme le tableau 6 permet de le retracer.

Tableau 6: Effectifs des flux migratoires entrants et sortants et solde migratoire entre 1987 et 2011 en milliers

\begin{tabular}{|c|c|c|c|c|c|c|c|c|c|c|c|c|c|}
\hline & 1987 & 1989 & 1991 & 1993 & 1995 & 1997 & 1999 & 2001 & 2003 & 2005 & 2007 & 2009 & 2011 \\
\hline Immi. & 17,2 & 26,7 & 33,3 & 34,7 & 31,2 & 44,5 & 48,9 & 59 & 60 & 84,6 & 109,5 & 57,3 & 42,3 \\
\hline Emi. & 40,2 & 70,6 & 35,3 & 35,1 & 33,1 & 25,3 & 31,5 & 26,2 & 29,3 & 29,4 & 42,2 & 65,1 & 76,4 \\
\hline Solde & -23 & $-43,9$ & -2 & $-0,4$ & $-1,9$ & 19,2 & 17,3 & 32,8 & 30,7 & 55,1 & 67,3 & $-7,8$ & $-34,1$ \\
\hline
\end{tabular}

Source : Population and Migration Estimates, CSO, 15 septembre 2011 [http://www.cso.ielen/media/csoie/releasespublications/documents/population/2011/Population, and,Migration,Estimates,April,2011.pdf]

Deux phases dans l'histoire récente des migrations irlandaises sont identifiées grâce au document (tableau 6). Depuis la fin des années 1980, l'immigration prend un essor d'abord progressif puis très marqué de 2003 à 2007; parallèlement, l'émigration recule après un pic en 1988/89, jusqu'à atteindre des valeurs moyennes basses au début des années 2000. Par ailleurs, après 2007, l'immigration ralentit brusquement et l'émigration, qui depuis 2003 donnait des signes de reprise, s'envole; le solde migratoire redevient négatif en 2008 après avoir été excédentaire treize années durant.

Ce tableau nous montre que, sur toute la période, aucun des flux ne se réduit au point de tarir : quand l'immigration prend de l'importance, l'émigration ne faiblit pas, en 2003 par exemple; inversement, l'immigration se maintient quand l'émigration a repris de la vigueur, en 2011 par exemple. Ainsi, dans l'Irlande du $\mathrm{xxI}^{\mathrm{e}}$ siècle, les migrations sont-elles nombreuses et en sens opposé. Le bilan démographique qui en résulte est avant tout positif. 
La population immigrée représente $12 \%$ de l'ensemble de la population de la République en 2011, soit environ 544000 personnes, dont $71 \%$ sont originaires de l'EU27 (58\% d'entre eux des pays de l'élargissement), $12 \%$ d'Asie et 7,6 \% d'Afrique; quinze ans plus tôt, les étrangers totalisaient à peine plus de $4 \%$ de la population ${ }^{4}$ de la République, $80 \%$ d'entre eux venus de l'UE15.

La présence de ces migrants venus d'horizons divers a affecté de manière notable certains indicateurs de la croissance démographique irlandaise. La structure par âge et par sexe de la population attire l'attention. Depuis l'arrivée des premiers immigrés, on enregistre en effet une forte présence dans les classes d'âge de la vie active et plus précisément chez les 25-39 ans, hommes comme femmes, pour la plupart migrants économiques. Leur nombre a ainsi renforcé la population des Irlandais issus du pic de naissance des années 1970-1980, ce qui fournit en partie l'explication de la légère sur-représentation de la classe des trentenaires, remarque faite au sujet du tableau 1. Entre 2006 et 2011, en terme d'âge encore, on a vu le nombre d'immigrés de plus de 65 ans augmenter de $27 \%$, celui d'enfants de moins de 14 ans de près de $50 \%$. Ces chiffres laissent penser que la nature de l'immigration a changé pour une part importante des étrangers venus initialement travailler dans la République. Nombreux sont ceux qui ont choisi depuis leur arrivée de s'installer avec leur famille à plus long terme, au titre du regroupement familial et bien que celui-ci ne soit pas généreusement accordé. Pour ce qui est de la répartition par sexe, on note une évolution qui rétablit un équilibre puisque, en 2006, près de $47 \%$ des immigrés étaient des hommes, pour $40,5 \%$ de femmes et $12,5 \%$ d'enfants et en $2011,42,6 \%$ d'hommes et $43 \%$ de femmes se sont installés en Irlande accompagnés de 14,4\% d'enfants. On peut en conclure que les déséquilibres en terme d'âge qu'ont pu entraîner la présence des premiers immigrants se rétabliront d'autant plus naturellement que les émigrants se situent en majorité dans les mêmes classes d'âge. Si les départs venaient à être plus nombreux, c'est l'accroissement de la population qui s'infléchirait. S'ils venaient à être plus déséquilibrés - aujourd'hui $53 \%$ des émigrants sont Irlandais - c'est la composition même de la population qui serait plus profondément marquée.

L'étude des convergences de la population irlandaise vers un modèle européen ne peut se limiter à l'analyse de la croissance de la population, de son relatif vieillissement et du jeu de ses migrations car une dimension plus sociétale s'impose. Les indicateurs présentés dans les paragraphes qui suivent permettent de donner la mesure des changements socio-démographiques en cours; ainsi l'évolution des valeurs maritales ou familiales, des croyances religieuses et le délicat

4. Chiffre obtenu à partir des données du recensement de 1996 relatives aux lieux de naissance, et dont ont été exclus les effectifs nord-Irlandais et la moitié des natifs d'Angleterre résidants en Irlande. Census 1996, Principal Demographic Results, CSO, Dublin, July 1997, 74 p., tableau 20, p. 57. 
sujet de l'appartenance ethnique sont-ils traités sucessivement afin de compléter le panorama proposé.

À la fois institution sociale et religieuse, longtemps rite de passage à la maturité et étape incontournable précédant la création d'une famille, le mariage est aujourd'hui un bon indicateur de l'évolution des mentalités, des comportements et du sentiment religieux. Le tableau 7 indique la fréquence de la pratique depuis 1980.

Tableau 7: Nombre de mariages et taux de nuptialité pour mille de 1980 à 2010

\begin{tabular}{|c|c|c|c|c|c|c|c|}
\hline Année & 1980 & 1985 & 1990 & 1996 & 2002 & 2008 & 2010 \\
\hline Nombre & 21792 & 18791 & 17838 & 16174 & 20556 & 22243 & 20635 \\
\hline$\%$ o & 5,1 & 4,5 & 4,5 & 5,2 & 5 & 5 & 4,6 \\
\hline
\end{tabular}

Sources: This was then this is now, p. 54, Statistical Yearbook 2011, p. 62, CSO, Dublin, October 2011, 351 p.

Le nombre de mariages en Irlande s'est maintenu à un niveau relativement stable au cours des trente dernières années et le taux de nuptialité de 2010, bien qu'inférieur à la valeur record de 7,5\%o enregistrée en 1970, reste comparable à la moyenne européenne de 4,5\% . La structure par âge de la population - sa sur-représentation des 25-35 ans - et la tradition catholique du pays peuvent en partie justifier un tel taux. Pourtant, comparativement à d'autres pays européens, catholiques et dotés d'une forte classe d'âge de jeunes adultes, le taux irlandais est relativement élevé. En effet, on relève en Espagne et en Italie, un taux de nuptialité de 3,6 \%o en 2010, 3,7 \%o au Portugal. Le nombre de mariages célébrés en Irlande s'est aussi maintenu grâce à deux facteurs apparus au cours de la dernière décennie : d'une part, le remariage est maintenant possible depuis que le divorce existe, sa législation a été introduite en 1996. Dans la population de la République en 2002, 21400 personnes étaient remariées, en 2010, deux fois plus (42960) de personnes avaient renoué des liens matrimoniaux. D'autre part, parmi les populations nouvellement installées sur le sol irlandais, le mariage est une pratique encore très courante, chez les Polonais par exemple, minorité nationale la plus représentée.

Le maintien du nombre de mariages célébrés au sein de la population s'accompagne pourtant de changements dont il convient de rendre compte ici. Lâge au mariage en est un. En Irlande, comme dans de nombreux pays européens, le mariage survient plus tardivement dans la vie : en 1980, la moyenne d'âge des Irlandais et Irlandaises au mariage était respectivement de 28,2 et 24,5 ans; en 2010 , ils ont 33,8 ans et elles ont 31,3 ans en moyenne. Le retardement de l'engagement matrimonial trouve, au début du XXI ${ }^{\mathrm{e}}$ siècle, un ensemble de causes. On peut citer la plus longue durée moyenne des études, la plus grande indépendance 
financière des jeunes femmes ou encore la meilleure acceptation du concubinage, chez les jeunes couples sans enfant en particulier. Ce sont ici autant de facteurs que la République partage avec ses voisins européens.

Un autre changement notable concerne la nature religieuse ou civile des mariages. Le tableau 8 retrace l'évolution du nombre et de la part des mariages civils depuis 1960 .

Tableau 8 : Mariages catholiques et civils et part des mariages civils sur totalité des mariages entre 1960 et 2008

\begin{tabular}{|c|c|c|c|c|c|c|c|c|c|}
\hline Année & 1960 & 1970 & 1980 & 1990 & 1996 & 2002 & 2004 & 2006 & 2008 \\
\hline Catholiques & 14866 & 19898 & 20974 & 16626 & 14607 & 15908 & 15978 & 16211 & 15978 \\
\hline Civils & 57 & 121 & 388 & 656 & 928 & 3683 & 4286 & 5127 & 5299 \\
\hline Taux Civils & $0,3 \%$ & $0,5 \%$ & $1,8 \%$ & $3,7 \%$ & $5,7 \%$ & $17,9 \%$ & $20,4 \%$ & $23 \%$ & $23,8 \%$ \\
\hline
\end{tabular}

Source : Statistical Yearbook 2002, tableau 3.9, p. 63, CSO, Dublin, October 2002, 415 p.

Rare dans les années 1960, le mariage civil a pris un essor remarquable à partir de la fin du $\mathrm{xx}^{\mathrm{e}}$ siècle et occupe une part qui semble s'être stabilisée depuis 2006. Certes, les trois quarts des mariages célébrés en Irlande demeurent catholiques et il s'agit d'une proportion considérable au regard d'autres pays catholiques européens et supérieure aux valeurs des pays les plus pratiquants que sont la Pologne et la Grèce. Pourtant la progression des mariages civils depuis 1996 révèle de profonds bouleversements dans le domaine des valeurs individuelles et elle est également à considérer dans un ensemble de données relatives à l'évolution du sentiment religieux. La question est abordée dans la section suivante, mais avant d'y venir, un second sujet d'étude lié à la sphère privée et visible dans l'organisation de la vie civile est celui des structures familiales.

Si les années 2010 se caractérisent dans la République par un nombre plus élevé de familles, avec un gain de $12 \%$ sur les cinq dernières années, et d'enfants, avec près de $10 \%$ supplémentaires sur la même période, le détail des situations familiales met en évidence de nombreux changements structuraux.

La diminution de la taille des familles irlandaises se poursuit, phénomène qui touche aussi la plupart des pays européens. Rapide entre 1991 et 2006, passant d'une moyenne de deux enfants à 1,4, elle est aujourd'hui stabilisée à 1,38 enfant, ce qui constitue une valeur inédite dans l'histoire de la famille irlandaise. Les naissances de rang 3 et au-delà ne forment plus qu'un nombre infime du total des naissances alors qu'elles étaient beaucoup plus courantes il y a trente ans. 
La diversité des configurations familiales est devenue plus manifeste au cours des quinze dernières années, comme en témoignent les données suivantes.

Tableau 9: Structure des cellules familiales en 1996 et 2011, effectifs et taux

\begin{tabular}{|l|r|c|r|c|c|}
\hline \multirow{2}{*}{\multicolumn{1}{|c|}{ Statut }} & \multicolumn{2}{|c|}{1996} & \multicolumn{2}{c|}{2011} & \multirow{2}{*}{ Variation 1996-2011 (\%) } \\
\cline { 2 - 5 } & Effectifs & Taux & Effectifs & Taux & \\
\hline Couples mariés sans enfant & 154854 & $(19,2)$ & 261652 & $(22,1)$ & $+106798(68,9)$ \\
\hline Concubins sans enfant & 18640 & $(2,3)$ & 83292 & $(7)$ & $+64652(346,8)$ \\
\hline Couple marié avec enfant(s) & 491567 & $(61)$ & 558682 & $(47,3)$ & $+67115(13,6)$ \\
\hline Concubins avec enfants & 12658 & $(1,5)$ & 60269 & $(5,1)$ & $+47611(376)$ \\
\hline Mère seule avec famille à charge & 108282 & $(13,4)$ & 186284 & $(15,8)$ & $+78002(72)$ \\
\hline Père seul avec famille à charge & 20834 & $(2,5)$ & 29031 & $(2,4)$ & $+8197(39,3)$ \\
\hline $\begin{array}{l}\text { Nombre total de cellules } \\
\text { familiales }\end{array}$ & 806835 & - & 1179210 & - & $+372375(46,2)$ \\
\hline
\end{tabular}

Sources : Données calculées à partir de Statistical Yearbook 2001 p. 15 et This is Ireland, tableau 14, p. 80

Le tableau 9 révèle d'une part la part grandissante du nombre de couples sans enfants : au total, ils représentaient $21,5 \%$ de l'ensemble des unités familiales en 1996, ils sont $29,1 \%$ en 2011. En effectifs, leur croissance est supérieure à celle de tous les parents réunis. Si les couples mariés sans enfants ont pris le plus d'essor, on note que le groupe des concubins sans enfant a progressé en nombre de manière comparable à celle des parents mariés. Inversement, les parents mariés ont vu leur proportion se réduire de $61 \%$ à $47,3 \%$ sur la même période tout en demeurant le modèle familial de loin le plus courant.

Ces tendances rendent manifeste une prise de distance relativement aux préceptes catholiques qui mettent encore en avant la fonction de procréation de l'union de l'homme et de la femme. Dans la société irlandaise du $\mathrm{xxI}^{\mathrm{e}}$ siècle, l'accès pour tous et toutes à des moyens de contraception a permis ces choix devenus individuels. D'autre part, les couples concubins avec enfants ont renforcé leur position mais demeurent le modèle familial choisi par le plus petit nombre de couples. La question très débattue est de savoir s'il s'agit d'un état transitoire ou définitif. Enfin, la progression des familles monoparentales est à souligner, elle est forte chez les femmes seules avec enfants à charge, qui répresentent $86 \%$ de cette population. Cette catégorie nous amène à conclure sur quelques remarques relatives aux statuts matrimoniaux.

L'émergence d'un statut de parent isolé et la reconnaissance suivie de la meilleure connaissance des familles monoparentales ont été possibles grâce aux avancées législatives en matière de divorce, et d'union civile plus récemment. La légalisation du divorce a rendu plus légitime les séparations qui existaient de 
facto dans la société. Malgré un nombre de cas non négligeable aujourd'hui et une forte poussée initiale due à un effet de rattrapage dans les premières années, le taux de divortialité demeure encore très bas en Irlande, 0,7 \%o, proche du taux italien de $0,9 \%$ mais très en deçà de la valeur moyenne de l'Union européenne de $2 \%$. Les détracteurs de la loi de 1996 craignaient qu'un chaos marital règne sur la République une fois le divorce rendu possible; leur peur ne s'est, à ce jour, pas matérialisée. Au contraire, la loi a donné l'occasion à un nombre croissant d'individus de reconstruire leur vie en couple, en se remariant notamment. Pour ce qui est du pacte civil, autre avancée législative répondant à la demande d'une partie de la population et modifiant la conception du statut marital, la République a fourni une réponse qui s'éloigne de ce que la religion dominante recommandait. En 2011, alors que les premiers pactes civils sont enregistrés, la République adopte une position progressiste comparativement à d'autres pays, historiquement plus tolérants mais qui ne se sont pas, à ce jour, engagés sur cette voix.

Lorganisation sociétale des hommes et des femmes d'Irlande a subi de nombreux bouleversements à l'instar de ce qui se passe dans les autres pays d'Europe : le choix de remettre le mariage à plus tard, voire celui de ne pas se marier, la plus grande précarité de la vie des couples et la diversité des interprétations de ce que vivre en couple signifie. À la très tardive introduction du divorce a fait suite la légalisation d'un pacte civil, preuve s'il en fallait que la République peut s'adapter aux réalités des nouveaux modes de vie.

S'interroger sur le changement démographique requiert aussi la prise en compte de la dimension identitaire des individus. L'indicateur de la religion est une des données identitaires recueillies lors du recensement. Cette information ne sera ici pas utilisée comme outil de mesure de la pratique ou du sentiment religieux mais comme signe de la diversité confessionnelle de l'Irlande du $\mathrm{xxI}^{\mathrm{e}}$ siècle, au même titre que l'appartenance ethnique de la section suivante permet de mieux appréhender la diversité culturelle. Dans les recensements, l'appartenance religieuse est plus précisément renseignée depuis 1991 dans l'énumération des fidèles de religions moins courantes.

S'il est une grande banalité d'associer la religion catholique à la République d'Irlande, il s'agit encore aujourd'hui d'une réalité, d'une part constitutionnelle ${ }^{5}$ et d'autre part reflétant l'identité religieuse de près de $85 \%$ de la population du pays. Le tableau 10 souligne cet état de fait tout en donnant une idée de l'appartenance confessionnelle des quelques $15 \%$ non catholiques.

5. Rappelons que la constitution de 1937 faisait ouvertement référence à la religion catholique (art. 44, sect. 2) comme seule religion de la nation et bien que la section ait été retirée depuis, le document reste encore aujourd'hui d'inspiration catholique. 
Tableau 10: Effectifs des principales confessions religieuses dans la République en 1991, 2006 et 2011

\begin{tabular}{|l|c|c|c|}
\hline & 1991 & 2006 & 2011 \\
\hline Catholiques (en milions) & 3,228 & 3,681 & 3,861 \\
\hline Eglise d'Irlande et Protestants & 95534 & 125585 & 134365 \\
\hline Musulmans & 3873 & 32539 & 49204 \\
\hline Orthodoxes, Coptes & 358 & 20798 & 45223 \\
\hline Autres chrétiens & 16329 & 29206 & 41161 \\
\hline Presbytériens & 13199 & 23546 & 24600 \\
\hline Apostoliques/Pentecôtistes & 285 & 8116 & 14043 \\
\hline Hindous & 953 & 6082 & 10688 \\
\hline Bouddhistes & 986 & 6516 & 8703 \\
\hline Méthodistes, Wesleyiens & 5037 & 12160 & 6842 \\
\hline
\end{tabular}

Sources: Statistical Yearbook 2001, tableau 1.9, p. 13 et This is Ireland 1, Census 2011, tableau 36, p. 104; CSO, Dublin, October 2001, 373 p.

Ces données rappelle la domination du catholicisme tout en montrant son recul dans l'Irlande contemporaine : en 1991, 91,5\% des Irlandais étaient catholiques, en 2006 , ils étaient $87,3 \%$ et $84,6 \%$ en 2011 . Les questions posées par le recul du catholicisme sont multiples : s'agit-il de désaffections, d'un effet de proportionnalité face à la montée d'autres confessions ou plus généralement du recul du religieux dans la société?

Le tableau ci-dessus fournit quelques éléments de réponse. Sur la période considérée, certaines confessions ont maintenu leur position proportionnellement aux autres, l'Église d'Irlande (3\%), le presbytérianisme (0,5\%), le méthodisme $(0,1 \%$ après un pic à $0,3 \%)$. L'attention est attirée par les confessions qui ont rallié un nombre relativement élevé de pratiquants au cours des quinze dernières années. Deux catégories peuvent être distinguées : les confessions chrétiennes et les autres.

Parmi les dénominations chrétiennes, toutes étaient présentes dans le paysage religieux du pays depuis plusieurs décennies et la part de leurs pratiquants, bien qu'ayant augmenté, demeure faible $(0,2$ à $0,3 \%)$. C'est le cas des pratiquants apostoliques, pentecôtistes et des autres chrétiens. L'essor de l'orthodoxie est plus remarquable. Les orthodoxes et coptes sont $1 \%$ de la population totale en 2011 alors que leur nombre était infime en 1991. L'arrivée de migrants européens suite à l'élargissement de l'Union en 2004 explique cet accroissement, le groupe des quelques 43000 pratiquants est à $80 \%$ constitué d'étrangers, dont près de neuf sur dix viennent de l'Europe de l'Est.

Les dénominations non-chrétiennes, islam, hindouisme et bouddhisme, sont plus remarquées dans ce paysage religieux resté si longtemps et exclusivement attaché à la chrétienté. Numériquement, l'islam affiche une progression semblable 
à celle de l'orthodoxie, avec une part passant de $0,1 \%$ à $1 \%$ des pratiquants en quinze ans. Pourtant, le groupe des mulsulmans diffère en cela qu'elle est plus également partagé entre Irlandais et étrangers puisque 38,4 \% des Musulmans pratiquants dans la République sont irlandais. Les musulmans qui ne sont pas irlandais sont majoritairement issus d'Asie et d'Afrique. Hindous et bouddhistes ont connu une évolution semblable, passant d'un nombre infime à respectivement $0,23 \%$ et $0,19 \%$ de l'ensemble de la population. Ces données montrent que le repli proportionnel du catholicisme est en partie imputable à la présence plus marquée de certaines religions qui demeurent cependant minoritaires dans le paysage religieux de la République.

L'éventuelle désaffection religieuse est plus difficile à mettre en évidence. Un indicateur fiable est la déclaration de non-appartenance religieuse recueillie lors des recensements. Le tableau 11 retrace l'évolution de cet indicateur au cours des cinquante dernières années, c'est-à-dire depuis qu'il est renseigné par le rencensement qui, par ailleurs, a toujours comporté un critère d'appartenance confessionnelle.

Tableau 11 : Nombre et pourcentage des personnes sans religion dans la République de 1961 à 2011

\begin{tabular}{|l|c|c|c|c|c|c|c|}
\hline & 1961 & 1971 & 1981 & 1991 & 2002 & 2006 & 2011 \\
\hline Sans religion & 1107 & 7616 & 39572 & 66270 & 138264 & 186318 & 269811 \\
\hline $\begin{array}{l}\text { Pourcentage de la } \\
\text { population totale }\end{array}$ & 0,04 & 0,25 & 1,5 & 1,87 & 3,53 & 4,4 & 5,96 \\
\hline
\end{tabular}

Sources : Statistical Yearbook 2001, tableau 1.9, p. 13 et This is Ireland 1, Census 2011, tableau 36, p. 104

Sur toute la période, le nombre de personnes déclarant ne pas avoir de religion est en en constante augmentation, une progression allant presque du simple au double sur les dix dernières années et sur des valeurs relativement élevées pour l'Irlande puisqu'il s'agit de près de $6 \%$ de la population interrogée. Les Irlandais forment la majeure partie de cette population, 60 à $70 \%$ selon les recencements depuis 2002, les Européens la zone géographique la plus représentée parmi les étrangers sans religion. Les proportions irlandaises sont certes faibles au regard de ce que la plupart des pays européens recensent ou estiment. Au Royaume-Uni, en 2001, la première mesure officielle de la population non-croyante s'élevait à $15 \%$ de la population totale et pourrait atteindre $40 \%$ pour le recensement de 2011; en France, selon les sources, les chiffres oscillent entre 20 et $40 \%$, de la population totale. En Irlande comme ailleurs, il est possible que les données soient sous-estimées, la confusion existant entre la tradition ou la culture religieuse, souvent héritée de la famille, et la pratique ou la croyance individuelle. Le profil 
plus détaillé des non-croyants laisse apparaître quelques points communs entre la République le reste de l'Union européenne : la classe d'âge la plus représentée est celle des adultes de 24 à 45 ans, $13 \%$ des 25-29 ans se disent sans religion en 2011 par exemple, et les hommes sont toujours plus nombreux que les femmes, les citadins plus nombreux que les ruraux. Notons enfin que parmi les indicateurs d'identité que les recensements irlandais recueillent, la religion est celui que le plus grand nombre refuse de renseigner. La réticence à définir l'appartenance religieuse est plus grande que celle relative au statut marital ou à la nationalité par exemple.

La part grandissante des personnes sans religion est à considérer en parallèle avec celle des athées. Leur nombre est en effet aussi en progression, de quelques centaines en 1991 à près de quatre mille en 2011.

Pour conclure, on soulignera que si la diversité confessionnelle s'est affirmée dans la République au cours de la dernière décennie, elle s'accompagne aussi de la volonté d'un nombre grandissant de vivre sans religion, qu'elle soit une pratique ou une simple appartenance culturelle. Le catholicisme demeure pourtant la religion déclarée d'une majorité approchant les $85 \%$, un chiffre élevé dans le contexte européen et même dans les pays de forte tradition catholique. Ajoutons enfin que les scandales relatifs aux comportements de certains membres du clergé catholique irlandaise dévoilés depuis quelques années peuvent avoir encouragé ou accéléré la désaffection enregistrée, bien qu’il ne soit pas encore établi dans quelle mesure ces événements sont liés au recul du catholicisme.

En matière de nationalité comme d'appartenance ethnique, les recensements irlandais abordent les questions aussi ouvertement que possible depuis que la diversité est devenue manifeste là où, dans d'autres pays européens, le recueil de données est interdit. L'introduction de l'indicateur de nationalité en 2002 et celui d'appartenance ethnique en 2006 permet de mieux cerner l'évolution de la composition de la population de la République, d'estimer dans quelle mesure elle est devenue multi-ethnique et quels enjeux se posent à elle. Certes, les critères ethniques utilisés dans les recensements sont hautement critiquables, trop vagues, ne définissant pas une ethnie liée à la culture, la langue ou un passé commun mais une nationalité, voire des critères raciaux; pourtant, ces seules données aujourd'hui disponibles font émerger l'altérité au-delà du seul critère de nationalité, ce qui, dans les années à venir, prendra son sens si les enfants des immigrants d'aujourd'hui deviennent irlandais. Le tableau 12 établit un premier profil à partir des données de 2006 et 2011. 
Tableau 12 : Catégories ethniques, part de la population totale et accroissement (en 2006) et 2011 en milliers

\begin{tabular}{|c|c|c|c|c|}
\hline Catégories & & Effectifs & $\begin{array}{l}\text { Part de la popu- } \\
\text { lation totale* }\end{array}$ & $\begin{array}{c}\text { Accroissement } \\
\text { 2006-2011* }\end{array}$ \\
\hline \multirow[t]{4}{*}{ Blancs } & & $(3956,6) 4264,4$ & $(94,8) 94,2$ & 7,78 \\
\hline & Irlandais & $(3645,2) 3822$ & $(87,4) 84,45$ & 4,8 \\
\hline & Gens du Voyage & $(22,3) 29,5$ & $(0,5) 0,65$ & 31,8 \\
\hline & Autres blancs & (289) 412,9 & $(6,9) 9,1$ & 42,8 \\
\hline \multicolumn{2}{|l|}{ Asiatiques } & $(52,3) 84,7$ & $(1,3) 1,87$ & 61,8 \\
\hline & Chinois & $(16,5) 17,8$ & $(0,4) 0,4$ & 7,8 \\
\hline & Autres Asiatiques & $(35,8) 66,8$ & $(0,9) 1,47$ & 86,7 \\
\hline \multicolumn{2}{|l|}{ Noirs } & $(44,3) 65$ & $(1,1) 1,44$ & 46,8 \\
\hline & Africains & $(40,5) 58,7$ & $(1,0) 1,3$ & 44,8 \\
\hline & Autres noirs & $(3,8) 6,3$ & $(0,1) 0,14$ & 68,2 \\
\hline \multirow[t]{2}{*}{ Autres/mixtes } & & (46.4) 40,7 & $(1,1) 0,9$ & $-12,3$ \\
\hline & Non renseigné & $(72,3) 70,3$ & $(1,7) 1,55$ & $-2,7$ \\
\hline \multicolumn{2}{|l|}{ Total } & $(4172) 4525$ & - & 8,4 \\
\hline
\end{tabular}

*En pourcentage

Source: CSO, tableau 1. A du volume 5, Census 2006, p. 11, CSO, Dublin, July 2007 et tableau 28, This is Ireland 1, Census 2011, p. 94, CSO, Dublin, March 2012

Les changements de composition de la population sont à la fois subtils et remarquables. Dans la population dite blanche, on note une faible variation entre 2006 et 2011 : ce groupe représente $94 \%$ de l'ensemble des personnes recensées, et le faible repli (six dizièmes) n'est pas significatif. Si les gens du voyage enregistrent une légère croissance, en partie dû à la vigoureuse natalité du groupe, elle demeure très marginale au sein de l'ensemble de la population irlandaise. En revanche, le groupe de culture irlandaise, qui occupe encore une écrasante majorité, recule proportionnellement, de près de trois points en cinq ans sous la poussée d'autres groupes. Le plus important numériquement est celui des Blancs non-irlandais, qui totalisent le plus fort accroissement dans cette catégorie et en effectifs, passant de 289000 à près de 213000 personnes entre 2006 et 2011. Lélargissement de l'Union européenne en 2004 est en grande partie responsable de cette montée et les autorités irlandaises ont encouragé le phénomène puisque le pays n'a pas imposé de restrictions transitoires de circulation pour les nouveaux Européens ${ }^{6}$. Il a au contraire facilité les démarches administratives et participé à l'organisation de foires au recrutement, en Pologne notamment. Loin d'être une immigration spontanée, l'arrivée de groupes de populations venus en nombre de

6. Le reste de l'Union, à l'exception du Royaume-Uni et du Danemark, a en effet imposé des mesures transitoires de restriction de circulation afin de maîtriser les flux migratoires des nouveaux membres. 
l'Europe de l'Est au moment des plus forts besoins en main-d'œuvre du Tigre Celtique s'est produite en grande partie sous le contrôle des agences de l'emploi national.

Les deux autres groupes recensés, populations asiatiques et noires, ont connu une croissance plus modérée au cours des cinq dernières années, avec des gains de 32,4 et 20,7 milliers d'individus respectivement. Pourtant, chacun de ces groupes représente dans l'ensemble de la population d'aujourd'hui une part au moins deux fois plus élevée que le groupe autochtone des gens du voyage. Au sein du groupe asiatique, la diversité domine : les Chinois représentent moins d'un tiers de la population recensée, et pourtant près d'un quart des ressortissants asiatiques, un groupe dont l'accroissement est peu marqué depuis 2006, comparable à celui de l'ensemble de l'ethnie blanche. Les autres Asiatiques ne forment pas nécessairement une population identifiable selon des critères d'ethnicité puisque formée de ressortissants philippins, indiens, pakistanais et malaisiens, pour les plus nombreux. La population dite noire se compose très majoritairement d'Africains, à $90 \%$, et les données relatives à la nationalité indiquent que les Nigérians sont les plus nombreux venus d'Afrique en Irlande ( $42 \%)$, suivis des Africains du Sud et des Mauriciens.

Le seul groupe à avoir reculé en nombre et en pourcentage de la population totale est celui des individus ne s'identifiant à aucune des trois autres catégories ou appartenant à plusieurs catégories. Leur repli est faible mais leur nombre nonnégligeable comparativement à l'ensemble. Les informations manquent pour comprendre cette catégorie et sa composition. Les détails des indicateurs de naissance et de l'origine ethnique des parents pourraient, par exemple, permettre de mesurer le brassage ethnique; ces données ne sont pas encore disponibles. Notons pour terminer l'analyse du tableau 11, que le nombre de personnes n'ayant pas renseigné l'indicateur est lui aussi en recul et lui aussi non-négligeable. Le recul du nombre d'individus peut s'interpréter comme le signe que les catégories sont mieux adaptées aux différentes situations ou que les données sont plus volontairement communiquées. La part non-négligeable, 1,55 \% de la population totale - une population plus grande que celle personnes se déclarant noires - rappelle qu'il s'agit de données sensibles, ni faciles à définir dans certains cas, ni divulguées avec aise dans d'autres.

Le tableau 12 montre à quel point la composition de la population irlandaise a entamé une importante mutation. Dans un pays qui n’a pas été une puissance coloniale, la diversité ethnique a, depuis le début du $\mathrm{xxI}^{\mathrm{e}}$ siècle, fait son entrée dans un paysage démographique qui reste néanmoins majoritairement blanc. L'impact de ce changement démographique sera encore plus visible à l'avenir si ces groupes conservent leurs traditions et leur altérité dans la société irlandaise. Dans le court terme et pour cantonner ces remarques au domaine de la démographie, 
il est possible d'aller au delà de la simple énumération à partir d'un dernier document présentant la structure par âge des groupes.

Tableau 13 : Structure par âge des groupes ethniques en 2011 en pourcentage

\begin{tabular}{|c|c|c|c|c|c|c|c|c|}
\hline Âge & Total & Irlandais & $\begin{array}{c}\text { Autres } \\
\text { blancs }\end{array}$ & Africains & $\begin{array}{c}\text { Autres } \\
\text { noirs }\end{array}$ & Chinois & $\begin{array}{c}\text { Autres } \\
\text { Asiatiques }\end{array}$ & Autres \\
\hline $0-9$ & 14,8 & 14,4 & 12 & 33,5 & 33 & 12,7 & 22,4 & 24,7 \\
\hline $10-19$ & 12,7 & 13,1 & 8,7 & 16,1 & 14,6 & 8 & 11 & 14,5 \\
\hline $20-29$ & 14,1 & 12,8 & 24 & 10,6 & 16,4 & 37,1 & 17,4 & 20,5 \\
\hline $30-39$ & 16,5 & 15 & 27,4 & 21,6 & 18,7 & 25,2 & 29,6 & 21,6 \\
\hline $40-49$ & 13,8 & 14 & 14 & 15,1 & 11,6 & 10,2 & 13,5 & 11 \\
\hline $50-59$ & 11,3 & 12,1 & 7,5 & 2,4 & 4 & 4,6 & 4,3 & 4,4 \\
\hline $60-69$ & 8,5 & 9,4 & 4 & 0,4 & 0,9 & 1,2 & 1 & 1,8 \\
\hline $70+$ & 8 & 8,9 & 2,3 & 0,1 & 0,7 & 0,6 & 0,4 & 1,2 \\
\hline
\end{tabular}

Source : Données calculées à partir du tableau 28, This is Ireland, p. 94, CSO, Dublin, March 2012

L'impact des groupes ethniques sur la structure de la population est manifeste dans les trois grandes classes d'âge, l'enfance, l'âge adulte et l'âge mûr. Deux groupes se distinguent pour ce qui est de la jeunesse : les populations noire et asiatique à l'exception des Chinois. Les 0-9 ans représentent un tiers des individus se déclarant appartenir au groupe de personnes dites noires et plus de deux fois la moyenne irlandaise; ils sont un peu plus d'un Asiatique sur cinq. Dans la même classe d'âge, Blancs non-irlandais et Chinois sont moins nombreux que la moyenne nationale.

Par ailleurs, les jeunes adultes de 20 à 39 ans composent la majeure partie des groupes ethniques, à l'exception des membres de la communauté noire qui sont le plus nombreux entre 30 et 49 ans. Si nombre d'entre eux sont des migrants économiques, il s'agit logiquement de l'âge où la mobilité est la plus grande. Enfin, dans tous les groupes, la proportion de la population de plus de soixante ans est faible, celle des autres Blancs est la moins faible mais n'équivaut qu’à la moitié du taux enregistré chez les Irlandais.

Ainsi, on comprend dans quelle mesure certaines populations, pour la majeure partie formées de non-nationaux, accentuent la jeunesse de la population irlandaise, tant dans les classes d'âge des plus jeunes que dans celle des trentenaires, comme la remarque a été faite précédemment. Les effets démographiques de la reprise de l'émigration de l'après-Tigre Celtique sont d'autant plus atténués que, contrairement à ce qui pouvait être attendu, une petite partie seulement des immigrés ont quitté la République depuis la récente crise économique et, nous l'avons souligné dans la section sur les migrations, l'immigration n’a pas cessé. 
D'autres phénomènes liés aux groupes ethniques sont apparus : les secondes générations d'enfants appartenants à plusieurs groupes ethniques par exemple, mais ne sont pas encore assez documentés à ce jour pour pouvoir établir un parallèle avec ce qui existe dans d'autres pays européens.

Les treize tableaux réunis ici et commentés rendent compte des profonds changements que connaît la population irlandaise depuis une quinzaine d'années et qui modifient sa composition de manière inédite. La population irlandaise du $\mathrm{XXI}^{\mathrm{e}}$ siècle partage maintenant plus de caractéristiques avec le reste des pays européens que par le passé. Le recul de la mortalité, l’allongement de l'espérance de vie, le relatif vieillissement de la population rapprochent en effet la République du profil de ses voisins européens. La fécondité encore élevée n'empêche pas l'évolution progressive des modèles traditionnels en matière de mariage, de vie maritale et de configuration familiale et laisse l'individu plus responsable de ses choix et à même de les mettre en oeuvre selon sa volonté. L'augmentation des séparations et des divorces ne s'accompagne pas de la baisse du nombre de mariages, de même que l'appartenance religieuse encore élevée mais plus variée se manifeste alors que la montée du sentiment non-religieux est de plus en plus visible. Les migrations en Irlande présentent, à ce jour, des caractéristiques peu communes à d'autres pays européens : la coexistence d'une immigration et d'une émigration redéfinit la composition de la population et contribue à souligner certains de ses traits, sa jeunesse dans le contexte européen notamment. Petit pays par le nombre d'habitants, huitième plus petit pays de l'Union Européenne qui représente à peine un pourcent du total de la population de cette zone géographique, la République connaît des mutations qui laissent penser que domine la convergence vers des caractéristiques européennes quand bien même certaines particularités démographiques et culturelles demeurent. 